\title{
Impact of Geophysical Fluids on UT1
}

\author{
Richard S. Gross \\ Jet Propulsion Laboratory, California Institute of Technology, Pasadena, CA, 91109 USA \\ email: Richard.Gross@jpl.nasa.gov
}

\begin{abstract}
Geophysical fluids have a major impact on the Earth's rotation. Tidal variations within the oceans are the predominant cause of subdaily length-of-day (lod) variations while those within the solid body of the Earth are a major source of longer period variations; tidal dissipation within the solid Earth and oceans cause a secular change in lod. Fluctuations of the atmospheric winds are the predominant cause of nontidal lod variations on sub-decadal time scales while decadal variations are caused by interactions between the fluid core and mantle.
\end{abstract}

Keywords. Earth, time

\section{Introduction}

The Earth's rotation changes on all observable time scales, from subdaily to decadal and longer, reflecting the wide variety of processes causing it to change. The changes in the Earth's rotation that are observed and the models that have been developed to explain them are reviewed here.

\section{Passive influence}

Changes in the Earth's rotation are usually studied by applying the principle of conservation of angular momentum. Under this principle, and in the absence of external torques, the rotation of the solid Earth changes when angular momentum is transferred between it and the fluid regions with which it is in contact. But by modifying the Earth's inertia the fluid regions of the Earth influence the Earth's rotation even when they are at rest and not exchanging angular momentum with the solid Earth. If the Earth is assumed to be axisymmetric and if the fluid core is assumed to be inviscid and otherwise decoupled from the mantle, then the core cannot respond to axial changes in the rotation of the mantle. The greatest principal moment of inertia of the Earth appropriate for studying changes in the axial rotation of the Earth is then not that of the entire Earth including core $C$ but is instead that of just the Earth's crust and mantle $C_{m}$. The presence of a passive fluid core thus has about an $11 \%$ effect on modeled lod variations.

\section{Tidal variations}

Tidal forces due to the gravitational attraction of the Sun, Moon, and planets deform the solid and fluid regions of the Earth causing the Earth's inertia tensor to change and thus causing the Earth's rotation to change. Tidal dissipation within the oceans and solid Earth causes the Earth's angular velocity and hence rotational angular momentum to decrease. By analyzing lunar and solar eclipse, lunar occultation, optical astrometric, and space-geodetic observations Morrison \& Stephenson (2001) found that the length-ofday has increased at an average rate of $+1.8 \pm 0.1$ millisecond/century (ms/cy) during the past 2700 years. Since the observed tidal acceleration of the Moon implies that the length-of-day should be changing by $+2.3 \mathrm{~ms} /$ cy due to tidal dissipation, some other mechanism must be acting to change the length-of-day by $-0.5 \mathrm{~ms} / \mathrm{cy}$, the most likely 
candidate being glacial isostatic adjustment. The isostatic adjustment of the solid Earth in response to the decreasing load on it following the last deglaciation causes the figure of the Earth to change, and hence causes the length-of-day to change.

Of the elastic solid, inelastic solid, and dynamic ocean responses of the Earth to tidal forces in the long-period tidal band, the elastic response of the solid Earth has the greatest effect on lod, with inelastic effects being only a few percent of the elastic, and with the effect of dynamic ocean tides being most important at the fortnightly tidal frequency. Fortunately, accurate models for the effect of elastic solid, inelastic solid, and dynamic ocean tides on lod are currently available (Gross 2009).

But in the diurnal and semidiurnal tidal bands the ocean tides have the greatest effect on lod. In fact, comparisons of observations with models show the dominant role that ocean tides play in causing subdaily UT1 and lod variations, with as much as $90 \%$ of the observed UT1 variance being explained by diurnal and semidiurnal ocean tides. Apart from errors in observations and models, the small difference that remains is probably due to nontidal atmospheric and oceanic effects (Chao et al. 1996).

\section{Nontidal variations}

Since 1830 the length-of-day has been observed to vary by up to $7 \mathrm{~ms}$ on decadal time scales. Such large variations are too great to be caused by atmospheric fluctuations. Even if the atmosphere's motion were to cease entirely, the resulting change in lod would be only about $3 \mathrm{~ms}$. So decadal variations in lod are thought to be primarily caused by core-mantle interactions. Under reasonable physical assumptions, surface observations of the secular variation of the magnetic field can be used to infer the motion of the fluid at the top of the core. Additional reasonable physical assumptions allow the angular momentum of the entire core to be computed from the inferred motion of the fluid at the top of the core. In fact, remarkably good agreement between the core angular momentum modeled in this manner and lod observations on decadal time scales is found, at least since 1900 (Ponsar et al. 2003).

Numerous studies have shown that atmospheric winds are the dominant cause of nontidal lod variations on time scales of a few days to a few years. For example, on intraseasonal time scales, the effect of atmospheric winds explains some $86 \%$ of the observed variance. Including the effect of surface pressure fluctuations increases the observed variance explained to $90 \%$ and additionally including oceanic effects further increases it to $92 \%$. Similar results are obtained on seasonal and interannual time scales (Gross et al. 2004).

\section{Acknowledgements}

The work described in this paper was performed at the Jet Propulsion Laboratory, California Institute of Technology, under contract with the National Aeronautics and Space Administration.

\section{References}

Chao, B. F., Ray, R. D., Gipson, J. M., Egbert, G. D., \& Ma, C. 1996, J. Geophys. Res. 101, 20151

Gross, R. S. 2009, J. Geodyn in press

Gross, R. S., Fukumori, I., Menemenlis, D., \& Gegout, P. 2004, J. Geophys. Res. 109, B01406

Morrison, L. V. \& Stephenson, F. R. 2001, J. Geodyn. 32, 247

Ponsar, S., Dehant, V., Holme, R., Jault D., Pais, A., \& Van Hoolst, T. 2003, in: V. Dehant, K. C. Creager, S. Karato, \& S. Zatman (eds.), Earth's Core: Dynamics, Structure, Rotation, Geodynamics Series (Washington DC: Amer. Geophys. Union), vol. 31, p. 251 